\title{
Effect of Microstructure on Dielectric Breakdown in Amorphous $\mathrm{HfO}_{2}$ Films
}

\author{
S.K. Nandi ${ }^{1,3,4}$, D. J. Llewellyn ${ }^{1,2}$, K. Belay ${ }^{1}$, D.K. Venkatachalam ${ }^{1}$, X. Liu ${ }^{1}$ and R.G. Elliman ${ }^{1}$ \\ 1. Department of Electronic Materials Engineering, Research School of Physics and Engineering, The \\ Australian National University, ACT-0200, Australia \\ 2. Centre for Advanced Microscopy, The Australian National University, ACT-0200, Australia \\ 3. Research School of Astronomy and Astrophysics, The Australian National University, ACT-0200, \\ Australia \\ 4. Department of Physics, The University of Chittagong, Chittagong-4331, Bangladesh
}

Hafnium-based oxides are currently among the leading candidates for resistive switching memory (ReRAM) because of their high performance, high scalability and reliability [1]. The physical and electrical properties of an oxide layer have a strong correlation with the microstructural properties of the metal electrodes in a metal-insulator-metal (MIM) structure [2,3]. The physical switching mechanism is mainly controlled by the interface between the oxide layer and the electrodes. Besides the bulk properties, the surface/interface roughness of the metal electrodes also has an effect on the electronic properties of thin-film MIM structures. Hence, it is imperative to gain a better understanding of the microstructure of the device structures to understand their physics of operation. In this study, we investigate the effect of the electrode/dielectric microstructure using atomic force microscopy (AFM) and high-resolution transmission electron microscopy (HRTEM). We show that the dielectric breakdown in $\mathrm{Pt} / \mathrm{HfO}_{2} / \mathrm{Ti} / \mathrm{Pt} \mathrm{MIM}$ structure has a strong dependence on the interface roughness of the electrode.

Fig. 1(a) shows a plan-view TEM image with selected area diffraction pattern of a $20 \mathrm{~nm}$ thick $\mathrm{HfO}_{2}$ film grown by atomic layer deposition (ALD) deposited on a Pt substrate, confirming the amorphous nature of the $\mathrm{HfO}_{2}$ film. Grazing incident-angle x-ray diffraction (GI-XRD) measurements performed on the same film confirmed the amorphous nature of as-deposited $\mathrm{HfO}_{2}$ (results not shown). The X-TEM image in Fig 1(b), confirms the thickness of layers in the device structure and highlights interface roughness associated with the underlying Pt electrode. Atomic force microscopy (AFM) in tapping mode was used to investigate the surface roughness of the Pt film, as shown in Fig. 2. Scans over $1 \mu \mathrm{m} \times$ $1 \mu \mathrm{m}, 2 \mu \mathrm{m} \times 2 \mu \mathrm{m}$ and $5 \mu \mathrm{m} \mathrm{x} \times 5 \mu \mathrm{m}$ were measured and root-mean-square (rms) roughness values were calculated for each scan. Fig. 2(a) and (b) show the topography of the bottom Pt electrode measured by atomic force microscopy (AFM) of the thinnest $(25 \mathrm{~nm})$ and the thickest $(150 \mathrm{~nm})$ films used in this study. It is evident that the $25 \mathrm{~nm}$ thick Pt film has a relatively smooth surface when compared to 150 $\mathrm{nm}$ films. The surface roughness of $\mathrm{HfO}_{2}$ is expected to be influenced by the roughness of the $\mathrm{Pt}$ electrode as ALD grown films grow conformally to the substrate surface. Fig. 2(c) and (d) display the topography of $20 \mathrm{~nm} \mathrm{HfO}_{2}$ on $25 \mathrm{~nm}$ and $150 \mathrm{~nm} \mathrm{Pt}$, respectively. Both $\mathrm{Pt}$ and $\mathrm{HfO}_{2}$ films were also deposited on $300 \mathrm{~nm}$ thermal oxide (nominally atomically smooth) substrates for comparison. Fig.2 (e) and (f) show the variation of rms roughness of $\mathrm{HfO}_{2}$ and $\mathrm{Pt}$ films as a function of their thickness, respectively. From the rms values, it is observed that the surface roughness of Pt film increase with film thickness. Fig. 2(g) shows that the breakdown voltage of the $20 \mathrm{~nm} \mathrm{HfO}_{2}$ as a function of the rms roughness of the bottom electrode, showing a clear reduction in breakdown voltage with increasing roughness. Simulations of the breakdown process suggest that this results partly from variations in film thickness with increasing film roughness and from electric field enhancement at electrode protrusions. 


\section{Acknowledgment:}

Dr. Simon Ruffell is acknowledged for the provision of XTEM services.

\section{References:}

[1] G. D. Wilk, R. M. Wallace and J. Anthony, Journal of Applied Physics 89 (10) (20101), p.5243.

[2] G. Bersuker, J. Yum, L. Vandelli, A. Padovani, L. Larcher, V. Iglesias, M. Porti, M. Nafría, K. McKenna and A. Shluger, Solid-State Electronics 65 (2011), p.146.

[3] K. Yew, D. Ang and G. Bersuker, IEEE electron device letters 33 (2) (2012), p.146.
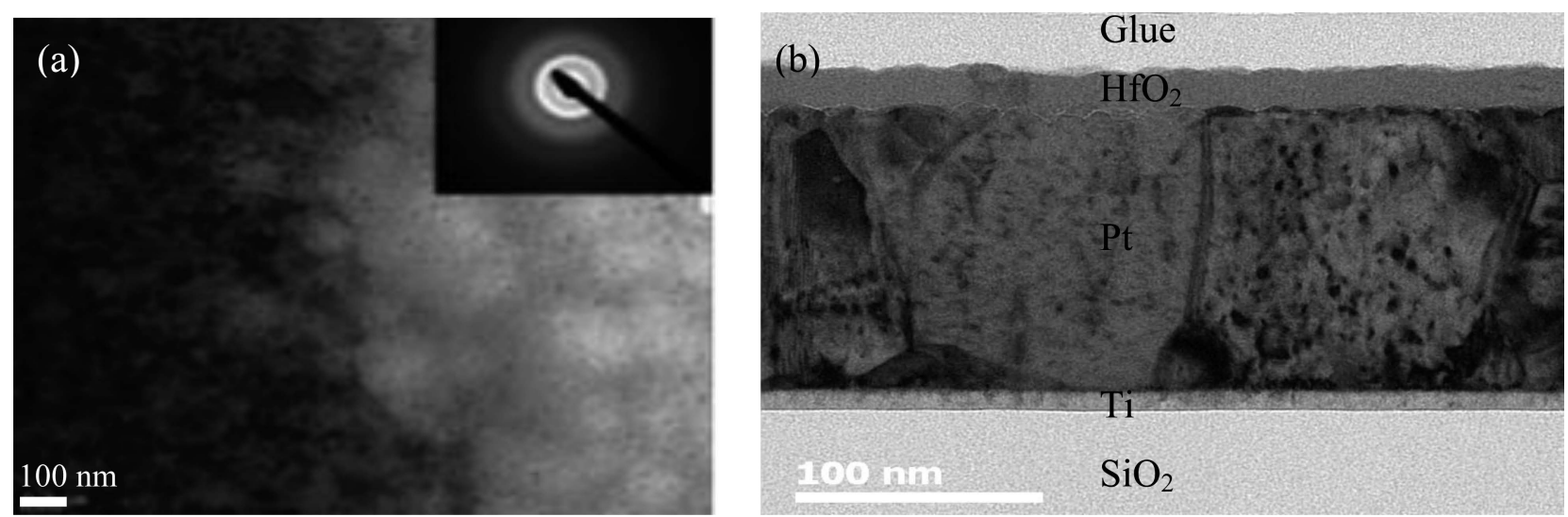

Figure 1: (a) Plan-view TEM image and SADP of as-deposited $20 \mathrm{~nm}$ thick $\mathrm{HfO}_{2}$ film grown by ALD; and (b) X-TEM image of an as-deposited $\mathrm{HfO}_{2}$ film on Pt showing interface roughness.
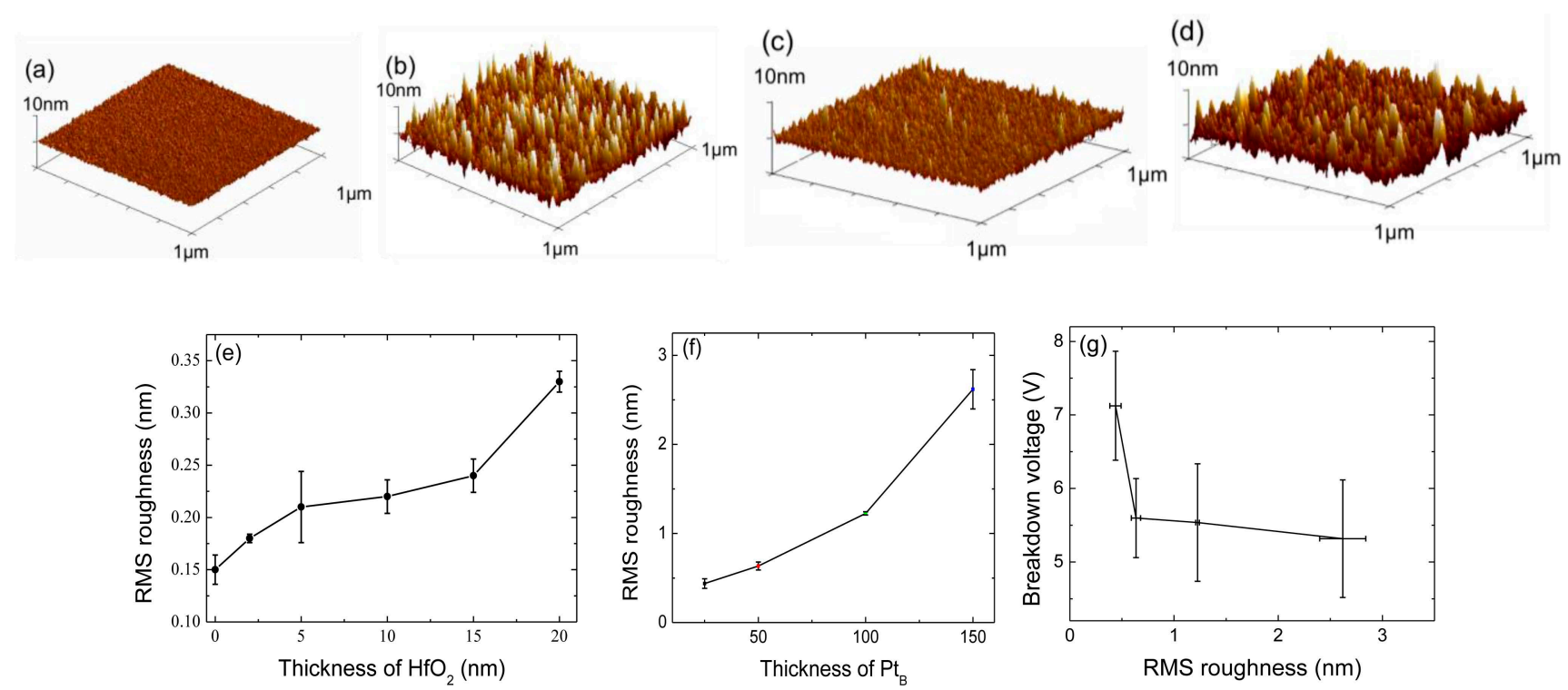

Figure 2: AFM topography images of (a) $25 \mathrm{~nm}$ and (b) $150 \mathrm{~nm} \mathrm{Pt}$; (c) $20 \mathrm{~nm} \mathrm{HfO}$ on $25 \mathrm{~nm} \mathrm{Pt}$ and (d) $20 \mathrm{~nm} \mathrm{HfO}_{2}$ on $150 \mathrm{~nm}$ Pt. Variation of rms roughness with (e) thickness of $\mathrm{HfO}_{2}$ on $300 \mathrm{~nm} \mathrm{SiO}_{2}$ and

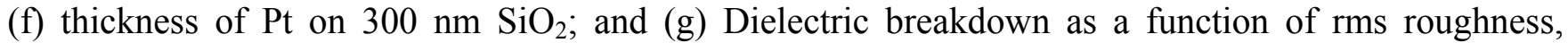
indicating correlation between the surface roughness and dielectric breakdown. 